Article

\title{
Synthesis, Characterization, and Antibacterial Activity of Cross-Linked Chitosan-Glutaraldehyde
}

\author{
Bin Li ${ }^{1,2}$, Chang-Lin Shan ${ }^{1}$, Qing Zhou ${ }^{1}$, Yuan Fang ${ }^{3}$, Yang-Li Wang ${ }^{2}$, Fei Xu ${ }^{4}$, \\ Li-Rong Han ${ }^{5, *}$, Muhammad Ibrahim ${ }^{1}$, Long-Biao Guo ${ }^{6, *}$, Guan-Lin Xie ${ }^{1}$ \\ and Guo-Chang Sun ${ }^{2, *}$
}

1 State Key Laboratory of Rice Biology, Institute of Biotechnology, Zhejiang University, Hangzhou 310058, China; E-Mails: libin0571@zju.edu.cn (B.L.); changlin_shan@163.com (C.-L.S.); zhou-0725-qing@163.com (Q.Z.); ibrahim@ciitsahiwal.edu.pk (M.I.); glxie@zju.edu.cn (G.-L.X.)

2 State Key Laboratory Breeding Base for Zhejiang Sustainable Plant Pest and Disease Control, Zhejiang Academy of Agricultural Sciences, Hangzhou 310021, China;

E-Mail:wangylaa@yahoo.com.cn

3 College of Chemistry and Life Sciences, Zhejiang Normal University, Jinhua 321004, China; E-Mail: Fy0579@zjnu.cn

4 Institute of Digital Agriculture, Zhejiang Academy of Agricultural Sciences, Hangzhou 310021, China; E-Mail: fxu@zju.edu.cn

5 Research and Development Center of Biorational Pesticides, Northwest A \& F University, Yangling, Shaanxi 712100, China

6 State Key Laboratory of Rice Biology, China National Rice Research Institute, Hangzhou 310006, China

* Authors to whom correspondence should be addressed; E-Mails: hlr4119@126.com (L.-R.H.); longbiaoguo@yahoo.com.cn (L.-B.G.); sungc01@sina.com (G.-C.S.); Tel.: +86-29-87092122 (L.-R.H.); +86-571-63370537 (L.-B.G.); +86-571-86404273 (G.-C.S.).

Received: 18 February 2013; in revised form: 17 April 2013 / Accepted: 26 April 2013 /

Published: 13 May 2013

\begin{abstract}
This present study deals with synthesis, characterization and antibacterial activity of cross-linked chitosan-glutaraldehyde. Results from this study indicated that cross-linked chitosan-glutaraldehyde markedly inhibited the growth of antibiotic-resistant Burkholderia cepacia complex regardless of bacterial species and incubation time while bacterial growth was unaffected by solid chitosan. Furthermore, high temperature treated cross-linked chitosan-glutaraldehyde showed strong antibacterial activity against the selected strain 0901 although the inhibitory effects varied with different temperatures. In addition,
\end{abstract}


physical-chemical and structural characterization revealed that the cross-linking of chitosan with glutaraldehyde resulted in a rougher surface morphology, a characteristic Fourier transform infrared (FTIR) band at $1559 \mathrm{~cm}^{-1}$, a specific X-ray diffraction peak centered at $2 \theta=15^{\circ}$, a lower contents of carbon, hydrogen and nitrogen, and a higher stability of glucose units compared to chitosan based on scanning electron microscopic observation, FTIR spectra, X-ray diffraction pattern, as well as elemental and thermo gravimetric analysis. Overall, this study indicated that cross-linked chitosan-glutaraldehyde is promising to be developed as a new antibacterial drug.

Keywords: antibacterial activity; characterization; chitosan; cross-link; glutaraldehyde

\section{Introduction}

The Burkholderia cepacia complex (Bcc) is a collection of genetically distinct but phenotypically similar bacteria that have emerged as life-threatening pulmonary pathogens in immunocompromised patients, particularly individuals with cystic fibrosis (CF). Indeed, the Bcc currently comprises 17 different species and infection with Bcc often leads to a fast decline in lung function and a markedly increased mortality [1-3]. The number of infections caused by the Bcc is increasing in China [4-6], while some species such as Burkholderia cenocepacia, Burkholderia contaminans, Burkholderia multivorans, Burkholderia seminalis, Burkholderia stabilis, and Burkholderia vietnamiensis have been isolated from agricultural and hospital environments in our previous studies [6-10].

Treatment of CF infections is very difficult due to the intrinsic resistance of Bcc bacteria to most clinically useful antibiotics, while some isolates of Bcc even can utilize penicillin $\mathrm{G}$ as a sole carbon source for growth [6,11]. Thus, it becomes important to identify newer and improved antibacterial therapies for CF patients. Recently, chitosan, a natural nontoxic biopolymer derived by deacetylation of chitin, a major component of the shells of crustacea such as crab, shrimp, and crawfish, has been applied in the fields of medicine, food, chemical engineering, pharmaceuticals, nutrition, environmental protection and agriculture [12,13]. In particular, chitosan not only has several advantages over other types of bactericides [14], but also has strong antibacterial activity against a variety of bacteria [15-20]. However, unfortunately, in our previous studies, chitosan solution possessed a limited antibacterial activity against Bcc bacteria [4,11].

Interestingly, previous studies have revealed that the biological activities of chitosan and its derivatives could be affected by different environments [14,21] and improved by either combining chitosan with different metal ions [22-25] or cross-linking chitosan with other organic compounds [26-28]. Indeed, chitosan has been cross-linked with glutaraldehyde in several studies [27,29], while these cross-linked complexes have been found to play a key role in the uptake of heavy metals [30,31]. However, to the best of our knowledge, little is known about the antibacterial activity of these cross-linked complexes against Bcc bacteria.

The aim of this study was to synthesize and characterize a cross-linked complex of chitosan and glutaraldehyde with strong anti-Bcc activity. 


\section{Results and Discussion}

Results from this study indicated that bacterial growth was unaffected by solid chitosan, but was strongly inhibited by the solid cross-linked chitosan-glutaraldehyde (CLCG) with cross-linking degree of $80.8 \%$ regardless of bacterial species and incubation time. In addition, high temperature treated CLCG showed strong antibacterial activity against the selected strain 0901 of the Bcc although the inhibitory effects varied with different temperatures. The differential antibacterial activity between CLCG and chitosan may be mainly due to the difference in their physical-chemical properties, which were evidenced by scanning electron microscopic (SEM) observation, Fourier transform infrared (FTIR) spectra, X-ray diffraction (XRD) pattern, as well as elemental and thermo gravimetric analysis. To the best of our knowledge, this study first synthesized the glutaraldehyde cross-linked chitosan with strong anti-Bcc activity.

\subsection{Antibacterial Activity of CLCG}

This study showed that no inhibition zones were observed when the nine Bcc strains were grown in the presence of solid chitosan in LB medium after 12, 24 and $48 \mathrm{~h}$ of incubation, indicating that solid chitosan used in this study has no antibacterial activity against these Bcc strains regardless of incubation time (Table 1). The non-inhibitory effect of chitosan may be due to the fact that chitosan is incapable to diffuse through the adjacent agar media when it is in a solid form [32]. Furthermore, this result is consistent with a number of previous studies that demonstrated chitosan particles would exhibit their potential in suppressing the bacterial growth only when they are in acidic media, in which the $\mathrm{NH}_{2}$ group in chitosan becomes a quaternary amino group and allows the chitosan to inhibit the growth of a variety of bacteria $[11,33,34]$.

Table 1. Antibacterial activity of chitosan and cross-linked chitosan-glutaraldehyde (CLCG) against Burkholderia cepacia complex after different incubation time.

\begin{tabular}{|c|c|c|c|}
\hline \multirow{2}{*}{ Treatments } & \multicolumn{3}{|c|}{ Inhibition diameter $(\mathrm{mm})$ after incubation of } \\
\hline & $12 \mathrm{~h}$ & $24 \mathrm{~h}$ & $48 \mathrm{~h}$ \\
\hline \multicolumn{4}{|c|}{ B. multivorans PW99 } \\
\hline Chitosan & $0.0 \pm 0.0 \mathrm{a}$ & $0.0 \pm 0.0 \mathrm{a}$ & $0.0 \pm 0.0 \mathrm{a}$ \\
\hline CLCG & $20.0 \pm 2.1 \mathrm{~d}$ & $19.8 \pm 2.1 \mathrm{~d}$ & $19.6 \pm 3.2 \mathrm{~d}$ \\
\hline \multicolumn{4}{|l|}{ B. stabilis $\mathrm{M} 8$} \\
\hline Chitosan & $0.0 \pm 0.0 \mathrm{a}$ & $0.0 \pm 0.0 \mathrm{a}$ & $0.0 \pm 0.0 \mathrm{a}$ \\
\hline CLCG & $17.6 \pm 1.5 \mathrm{c}$ & $17.5 \pm 1.5 \mathrm{c}$ & $17.3 \pm 1.5 \mathrm{c}$ \\
\hline \multicolumn{4}{|c|}{ B. seminalis $\mathrm{R} 456$} \\
\hline Chitosan & $0.0 \pm 0.0 \mathrm{a}$ & $0.0 \pm 0.0 \mathrm{a}$ & $0.0 \pm 0.0 \mathrm{a}$ \\
\hline CLCG & $15.5 \pm 1.5 \mathrm{~b}$ & $15.3 \pm 1.4 \mathrm{~b}$ & $15.0 \pm 1.6 \mathrm{~b}$ \\
\hline \multicolumn{4}{|l|}{ B. seminalis $\mathrm{S} 9$} \\
\hline Chitosan & $0.0 \pm 0.0 \mathrm{a}$ & $0.0 \pm 0.0 \mathrm{a}$ & $0.0 \pm 0.0 \mathrm{a}$ \\
\hline CLCG & $16.5 \pm 1.6 \mathrm{bc}$ & $16.3 \pm 1.6 \mathrm{bc}$ & $16.1 \pm 2.0 \mathrm{bc}$ \\
\hline \multicolumn{4}{|c|}{ B. vietnamiensis $\mathrm{S} 23$} \\
\hline Chitosan & $0.0 \pm 0.0 \mathrm{a}$ & $0.0 \pm 0.0 \mathrm{a}$ & $0.0 \pm 0.0 \mathrm{a}$ \\
\hline CLCG & $16.6 \pm 0.7 \mathrm{bc}$ & $16.4 \pm 0.9 \mathrm{bc}$ & $16.1 \pm 0.8 \mathrm{bc}$ \\
\hline
\end{tabular}


Table 1. Cont.

\begin{tabular}{|c|c|c|c|}
\hline \multicolumn{4}{|c|}{ B. contaminans $\mathrm{Y} 4$} \\
\hline Chitosan & $0.0 \pm 0.0 \mathrm{a}$ & $0.0 \pm 0.0 \mathrm{a}$ & $0.0 \pm 0.0 \mathrm{a}$ \\
\hline CLCG & $24.0 \pm 1.2 \mathrm{~g}$ & $23.5 \pm 1.4 \mathrm{f}$ & $23.4 \pm 1.6 \mathrm{f}$ \\
\hline \multicolumn{4}{|c|}{ B. cenocepacia Y8 } \\
\hline Chitosan & $0.0 \pm 0.0 \mathrm{a}$ & $0.0 \pm 0.0 \mathrm{a}$ & $0.0 \pm 0.0 \mathrm{a}$ \\
\hline CLCG & $22.6 \pm 2.0 \mathrm{f}$ & $22.3 \pm 2.0 \mathrm{ef}$ & $22.1 \pm 2.2 \mathrm{e}$ \\
\hline \multicolumn{4}{|c|}{ B. cenocepacia $\mathrm{Y} 17$} \\
\hline Chitosan & $0.0 \pm 0.0 \mathrm{a}$ & $0.0 \pm 0.0 \mathrm{a}$ & $0.0 \pm 0.0 \mathrm{a}$ \\
\hline CLCG & $20.3 \pm 1.6 \mathrm{de}$ & $20.0 \pm 1.5 \mathrm{~d}$ & $19.9 \pm 1.7 \mathrm{~d}$ \\
\hline \multicolumn{4}{|c|}{ B. seminalis 0901} \\
\hline Chitosan & $0.0 \pm 0.0 \mathrm{a}$ & $0.0 \pm 0.0 \mathrm{a}$ & $0.0 \pm 0.0 \mathrm{a}$ \\
\hline CLCG & $21.3 \pm 2.7 \mathrm{e}$ & $21.0 \pm 2.9 \mathrm{de}$ & $20.9 \pm 2.9 \mathrm{de}$ \\
\hline
\end{tabular}

Data were pooled from two independent experiments and shown as means \pm standard error. Means in a column followed by the same letter are not significantly different $(p<0.05)$.

In contrast with the result of chitosan, clear inhibitory zones were produced by CLCG, while the inhibition zone diameter of CLCG against the nine Bcc strains is significantly greater than the corresponding that of chitosan after 12, 24 and $48 \mathrm{~h}$ of incubation (Table 1). This result revealed that CLCG markedly suppressed the growth of the nine Bcc strains regardless of bacterial species and incubation time. Furthermore, this result in this study is generally consistent with the result of several previous studies, which showed that crosslinking by glutaric dialdehyde might aggravate the inhibitory effect of chitosan against some bacteria such as Escherichia coli and Bacillus subtilis [35,36]. In addition, Mcconnell et al. [37] reported that noncrosslinked chitosan films were digested by both pancreatic and colonic enzymes produced by the human colonic bacteria, while glutaraldehyde crosslinked chitosan films were resistant to both enzyme systems. Therefore, it could be suggested that CLCG might be able to be used as a promising novel marine antibacterial agent.

In general, the diameter of the inhibition zone for CLCG against the nine Bcc strains was very slightly reduced with the increase of incubation time, indicating that the antibacterial activity of CLCG was unaffected by incubation time. In contrast, the difference in inhibition zone diameter was noted between Bcc species. Indeed, the maximum inhibition zone diameter of 24.0, 23.5 and $23.4 \mathrm{~mm}$ was reached by B. contaminans strain Y4, while the minimum inhibition zone diameter of 15.5, 15.3 and $15.0 \mathrm{~mm}$ was obtained by $B$. seminalis strain R456 after 12, 24 and $48 \mathrm{~h}$ of incubation, respectively (Table 1). This result revealed the differential sensitivity of Bcc species to CLCG. However, compared to chitosan, CLCG showed stronger antibacterial activity against the nine Bcc strains regardless of bacterial species (Table 1).

In addition to the difference in CLCG sensitivity among the Bcc species, strains within a Bcc species also exhibited differential sensitivity to CLCG. Indeed, there was no significant difference in the diameter of the inhibition zone between strain R456 and S9 of B. seminalis regardless of incubation time. In contrast, the diameter of the inhibition zone of $B$. cenocepacia strain Y8 is significantly greater than that of B. cenocepacia strain Y17 regardless of incubation time (Table 1). The difference in the sensitivity of Bcc strains to CLCG may be attributed to the complex interaction between CLCG and Bcc 
bacteria. However, the growth of all tested Bcc strains was significantly inhibited by CLCG compared to chitosan (Table 1).

This study also indicated that no inhibition zone was observed when the selected strain 0901 of the Bcc was grown in the presence of heat treated chitosan in LB medium, indicating that solid chitosan has no antibacterial activity against Bcc strain 0901 even if after different heat treatment (Figure 1). In contrast, CLCG treated at $20^{\circ} \mathrm{C}, 40^{\circ} \mathrm{C}$ and $70{ }^{\circ} \mathrm{C}$ produced clear inhibitory zones. Indeed, the inhibition zone diameter of CLCG that treated at $20^{\circ} \mathrm{C}$ for $2 \mathrm{~h}$ was $16.5 \mathrm{~mm}$. When CLCG was treated at $40{ }^{\circ} \mathrm{C}$ and $70{ }^{\circ} \mathrm{C}$ for $2 \mathrm{~h}$, the inhibition zone diameter was significantly reduced by $30.4 \%$ and $35.6 \%$, respectively, compared to that of CLCG treated at $20{ }^{\circ} \mathrm{C}$. When heat treated temperature was $100{ }^{\circ} \mathrm{C}$, the inhibition zone diameter of CLCG was increased by $45.6 \%$ compared to that of CLCG treated at $20^{\circ} \mathrm{C}$ (Figure 1). Although the antibacterial activity of CLCG against Bcc strain 0901 would show different when CLCG was treated at different temperature, this result clearly indicated that heat treated CLCG had stronger antibacterial activity against Bcc strain 0901 compared to the corresponding heat treated chitosan regardless of the heat treated temperature.

Figure 1. Effect of temperature on antibacterial activity of chitosan and cross-linked chitosan-glutaraldehyde (CLCG) against Burkholderia seminalis strain 0901. Data from the repeated experiment were pooled and subjected to analysis of variance. Columns with the same letters are not significantly different $(p<0.05)$. Error bars represent the standard error of the mean.

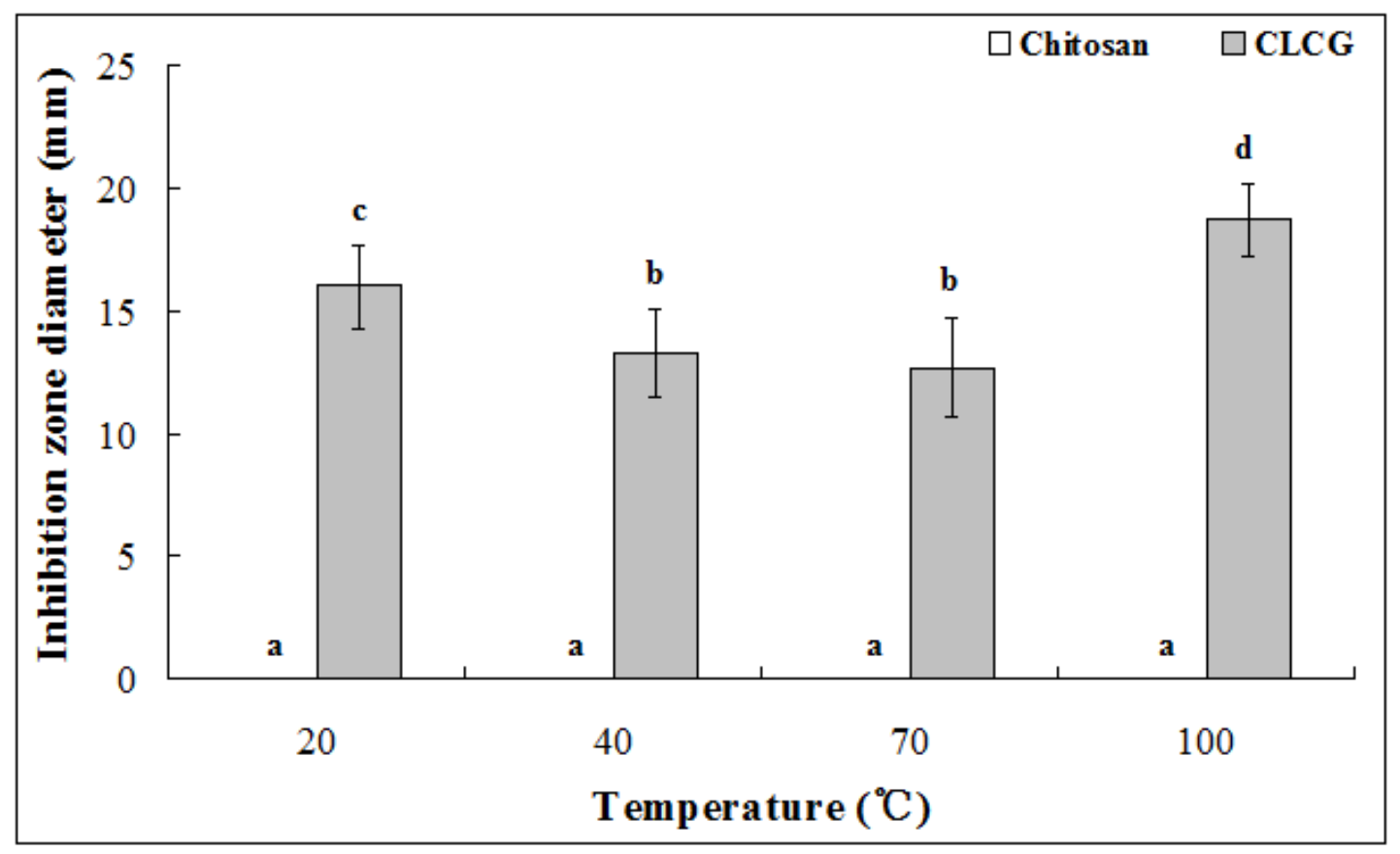

The result from this study also revealed that the antibacterial activity of CLCG may be mainly from itself, but not from either chitosan or glutaraldehyde. Indeed, the effect of chitosan could be easily excluded because solid chitosan used in this study shows no antibacterial activity against the Bcc strains. On the other hand, it is well known that $2 \%$ alkaline glutaraldehyde is the most widely used disinfectant. However, the risk of glutaraldehyde contamination has been markedly reduced by removing the non cross-linked glutaraldehyde from air dry CLCG sample with double distilled water. Furthermore, the 
weak acid chitosan solution in this study may abolish the antibacterial effect of glutaraldehyde for the glutaraldehyde activity at low concentrations is favored by an alkaline $\mathrm{pH}$. In addition, the diameter of the inhibition zone for CLCG against Bcc strain 0901 was significantly affected by heat treatment, indicating that the antibacterial activity of CLCG mainly depends on the crosslink reaction between chitosan and glutaraldehyde.

The interaction between chitosan with glutaraldehyde have received considerable attention over the past few decades [35,38], while the obtained data from ${ }^{13} \mathrm{C}$ NMR, infrared and Raman spectroscopies evidenced the formation of an ethylenic double bond in the chitosan-glutaraldehyde interaction [39]. In addition, two main crosslinking mechanisms, involving formation of Schiff's base structures or Michael-type adducts, have been proposed for the reaction of chitosan and glutaraldehyde. However, the nature of the chemical bonding between chitosan amine groups and glutaraldehyde has been subject of controversy, which may be due to this reason that the rate of chemical gelation of chitosan chains with glutaraldehyde depends on various parameters, namely $\mathrm{pH}$, ionic strength, temperature, chitosan concentration and degree of crosslinking $[38,40]$.

This result indicated that the inhibition in bacterial growth should be mainly due to the direct in vitro antibacterial activity of CLCG. However, Zhang et al. [35] found that in addition to increased antibacterial activity, glutaric dialdehyde crosslinking also enhanced chitosan uptake on the surface of cotton fabrics, with good durability of antibacterial properties to washing. This may be due to that glutaric dialdehyde is a binary aldehyde compound. One aldehyde group of glutaraldehyde reacts with amino group of chitosan, contributing to the antibacterial activity, while the other reacts with cellulose of cotton fabrics, improving the fastness property. Therefore, it could be expected that crosslinking by glutaraldehyde may have a direct and indirect contribution to the antibacterial activity of CLCG.

\subsection{Cross-Linking Degree of CLCG}

Results from this study indicated that the cross-linking degree of the synthesized CLCG with strong anti-Bcc activity was $80.8 \%$, while the other synthesized products with different cross-linking degrees were discarded for limited anti-Bcc activities (data not shown). This result is in agreement with the result of previous studies [41-43], which reported that in addition to degree of deacetylation in chitosan, the degree of crosslinking also controls the properties of chitosan. In addition, a number of studies have revealed that the aldehyde groups react immediately with the $-\mathrm{NH}_{2}$ groups along the chitosan chains after introducing wet chitosan microspheres into glutaraldehyde solution $[29,35,38,42,44,45]$. Therefore, it could be suggested that CLCG might be formed by cross-linking chitosan with glutaraldehyde at amino groups.

\subsection{SEM of CLCG}

Figure 2 presents the SEM micrographs illustrating the surface morphology of CLCG at four different $(1500 \times, 500 \times, 250 \times$ and $200 \times)$ magnifications. In general, this result clearly indicated that there is a difference in the surface microscopic morphology between chitosan and CLCG. Indeed, compared to the smooth, dense and flat morphology of chitosan [46], the SEM images in this study revealed that CLCG had a rough and porous surface (Figure 2), which is consistent with the result of previous studies [29,35,47]. In contrast, Wang et al. [48] and Kulkarni et al. [49] revealed that the microspheres of 
glutaraldehyde-crosslinked chitosan were spherical and have a smooth outer structure. Furthermore, Fang and $\mathrm{Hu}$ [50] found that the ultrastructure of the glutaraldehyde-crosslinked chitosan is a uniform and cloud-like. These differential results should be attributed to the fact that cross-linking degree and degree of deacetylation influences the size and morphology of glutaraldehyde-crosslinked chitosan [47,51].

Figure 2. SEM images of cross-linked chitosan-glutaraldehyde (CLCG) at four different magnifications (a) 1500×; (b) 500×; (c) 250×; and (d) 200×.
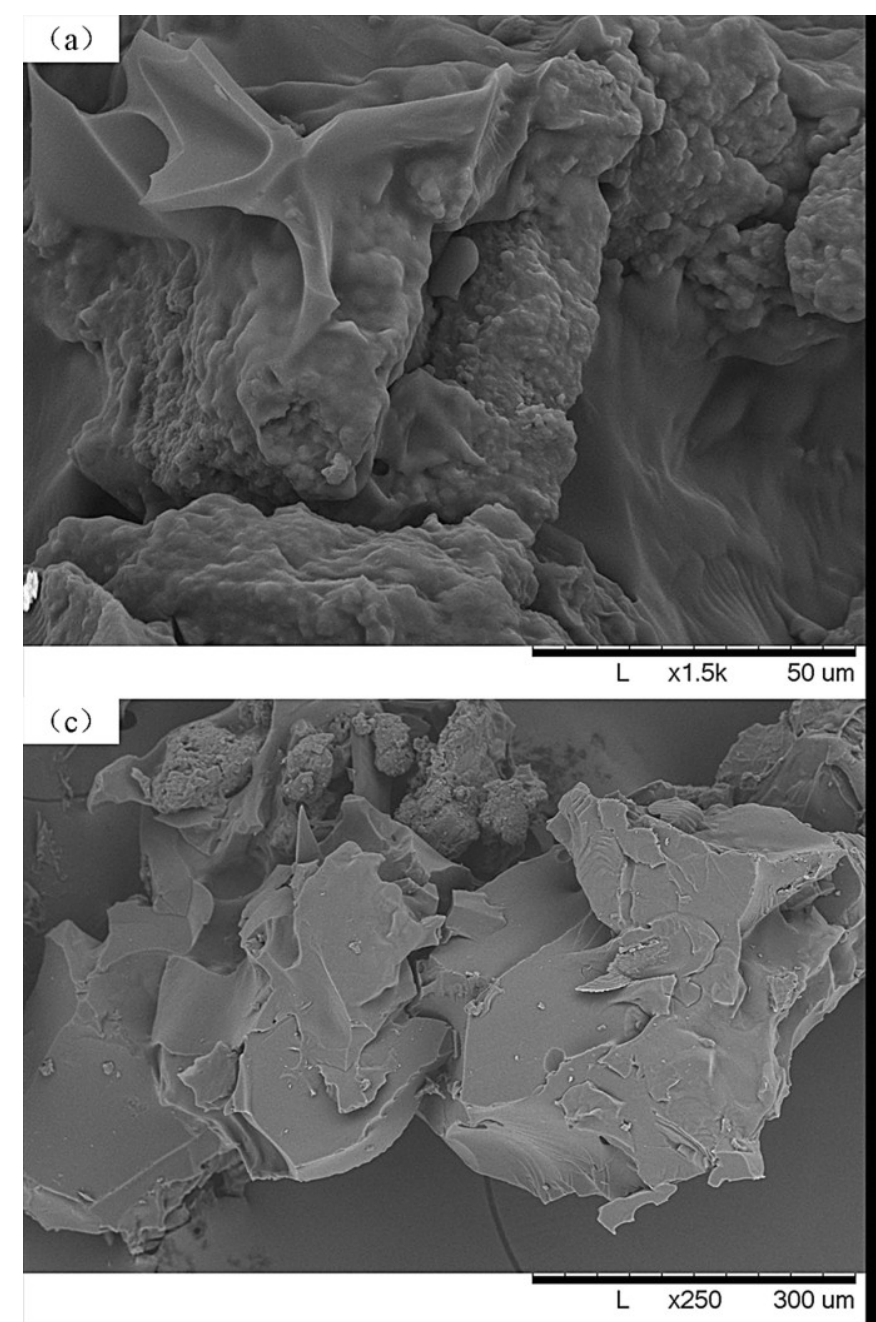

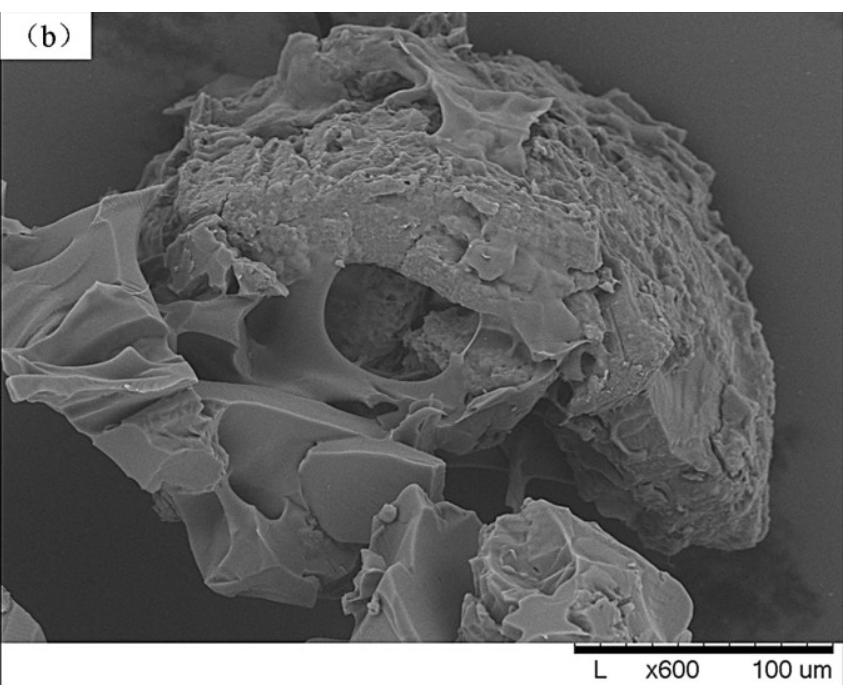

(d)

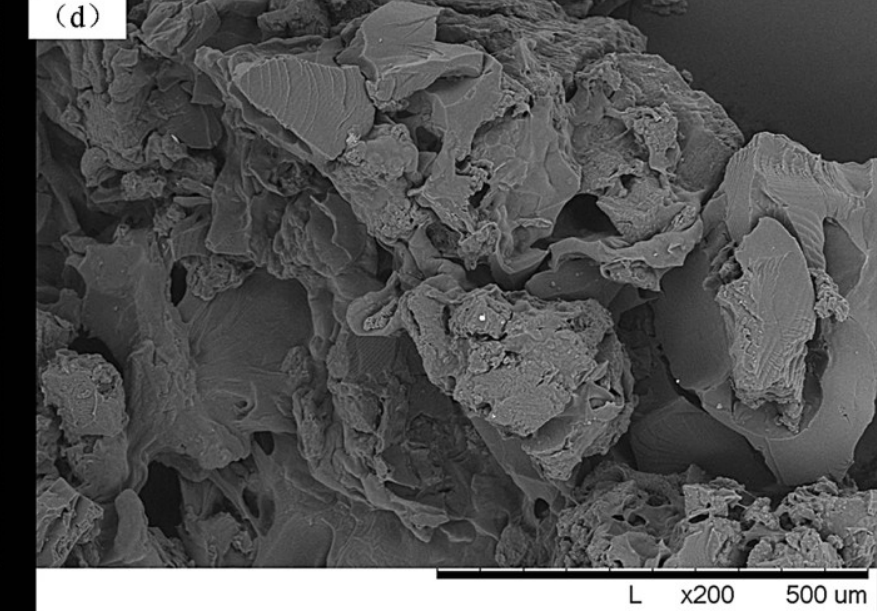

The rough surface morphology may be due to the insufficient cross-linking of CLCG or the glutaraldehyde groups partly grafted on chitosan, indicating that the reaction has taken place on the surface. Furthermore, the porous structure of CLCG in this study may offer more adsorption sites for adsorbate, which generally supported the fact that glutaraldehyde-crosslinked chitosan has been widely applied in the uptake of heavy metals [30,31] and drug delivery [51]. In addition, CLCG with a higher total surface area and a more open pore structure could be supposed to be favorable to interact with the Bcc strains, which may at least partially explain this result that the growth of the Bcc strains were significantly inhibited by CLCG, but not solid chitosan. 


\subsection{FITR Spectrum of CLCG}

FTIR spectra of chitosan and CLCG were displayed in Figure 3a,b, respectively. The main peaks for chitosan can be assigned as follows: $3439 \mathrm{~cm}^{-1}$ (N-H and O-H stretching vibration), $2925 \mathrm{~cm}^{-1}$ $\left(\mathrm{CH}_{3}\right.$ symmetric stretch), $1666 \mathrm{~cm}^{-1}$ ( $\mathrm{C}=\mathrm{O}$ stretching vibration), $1438 \mathrm{~cm}^{-1}$ ( $\mathrm{C}-\mathrm{N}$ stretching vibration), $1363 \mathrm{~cm}^{-1}$ ( $\mathrm{CH}_{3}$ bending vibration), $1155 \mathrm{~cm}^{-1}$ (C-O-C bending vibration), and $1073 \mathrm{~cm}^{-1}(\mathrm{C}-\mathrm{OH}$ stretching vibration). However, some major changes have been observed in the spectrum of CLCG by comparing the spectral differences in the $4000-500 \mathrm{~cm}^{-1}$ region of FTIR spectra between chitosan and CLCG. The FTIR spectrum of CLCG revealed that the N-H and O-H stretching vibration at $3439 \mathrm{~cm}^{-1}$ shifts to $3417 \mathrm{~cm}^{-1}$, the $\mathrm{CH}_{3}$ symmetric stretch at $2925 \mathrm{~cm}^{-1}$ shifts to $2937 \mathrm{~cm}^{-1}$, the $\mathrm{C}=\mathrm{O}$ stretching vibration at $1666 \mathrm{~cm}^{-1}$ shifts to $1645 \mathrm{~cm}^{-1}$, the $\mathrm{C}-\mathrm{N}$ stretching vibration at $1438 \mathrm{~cm}^{-1}$ shifts to $1406 \mathrm{~cm}^{-1}$, and the $\mathrm{C}-\mathrm{OH}$ stretching vibration at $1073 \mathrm{~cm}^{-1}$ shifts to $1037 \mathrm{~cm}^{-1}$ (Figure 3). Furthermore, the $\mathrm{CH}_{3}$ bending vibration at $1363 \mathrm{~cm}^{-1}$ and $\mathrm{C}-\mathrm{O}-\mathrm{C}$ bending vibration at $1155 \mathrm{~cm}^{-1}$ were observed in FITR of chitosan, but not in FITR of CLCG. In contrast, the band at $1559 \mathrm{~cm}^{-1}$ (amide II) was found in FITR of CLCG, but not in FITR of chitosan (Figure 3).

Figure 3. FTIR spectra of (a) chitosan and (b) cross-linked chitosan-glutaraldehyde (CLCG).

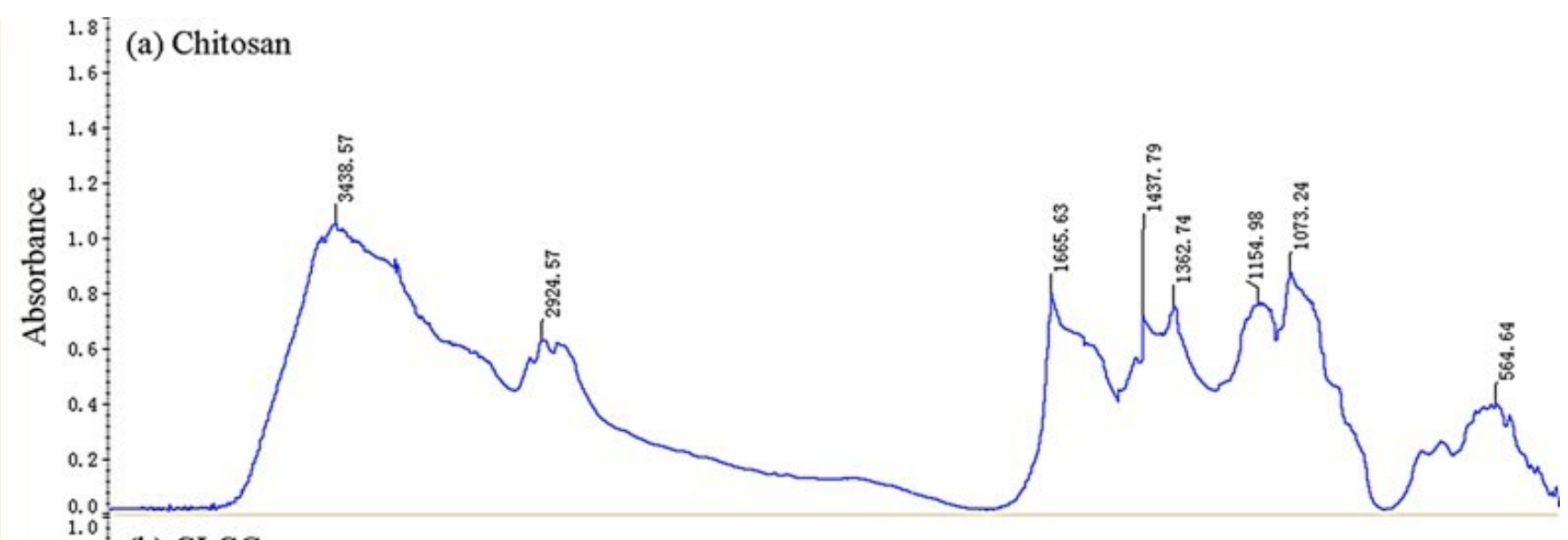

(b) CLCG

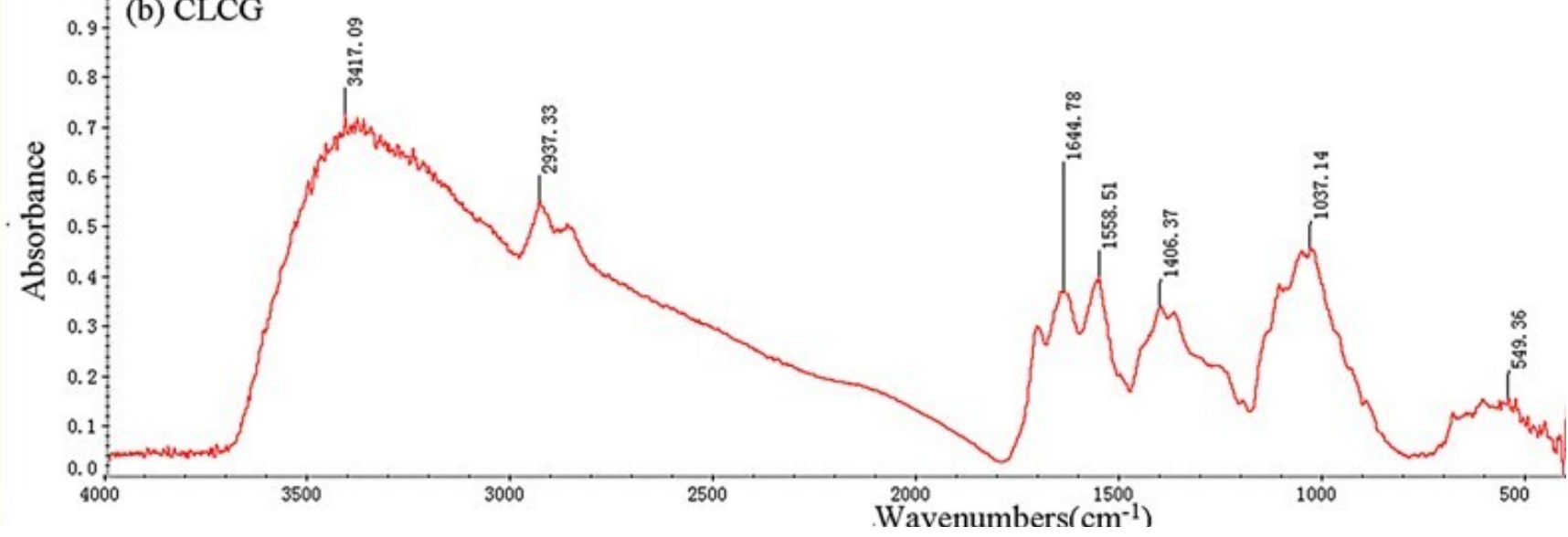

The result from this study is consistent with many previous studies [26,29,52], which have demonstrated that there was a difference in FITR profile between chitosan and CLCG. The peaks of chitosan at $1363 \mathrm{~cm}^{-1}$ and $1155 \mathrm{~cm}^{-1}$ disappeared, indicating that the two peaks may be hindered by glutaraldehyde cross-linked structure of chitosan. The appearance of the peak at $1559 \mathrm{~cm}^{-1} \mathrm{can}$ be 
attributed to the crosslinking reaction of chitosan and glutaraldehyde. Indeed, Ramachandran et al. [53] found that the new sharp peak at $1610 \mathrm{~cm}^{-1}$ represents stretching vibrations of $\mathrm{C}=\mathrm{N}$ in Schiff's base formed by the reaction of glutaraldehyde and chitosan. Furthermore, Knaul et al. [45] found that the chitosan film that reacted with glutaraldehyde exhibits a strong absorbance at $1664 \mathrm{~cm}^{-1}$, while Gupta and Jabrail [41] reported that the IR spectra have shown a strong absorption band at $1660 \mathrm{~cm}^{-1}$ in the glutaraldehyde cross-linked chitosan microsphere. In addition, Oyrton et al. [39] revealed that the increase of glutaraldehyde in the sequence of these modified chitosans caused a successive increase in intensity of ethylenic bond frequency at $1562 \mathrm{~cm}^{-1}$.

The result from this study indicated that the amide II band at $1559 \mathrm{~cm}^{-1}$ that may contribute to the amine- $\mathrm{NH}_{2}$ group was specific for CLCG. Interestingly, Tripathi et al. [32] revealed the change in the characteristic shape of the chitosan spectrum after cross-linking while stretching vibration spectra of the amide group of chitosan-based antimicrobial films appear at $1560 \mathrm{~cm}^{-1}$. Furthermore, Zhang et al. [35] reported that the intensity at 1557.19 reveals the quaternary-amino band. Thus, the antibacterial activity of CLCG may be due to the positive charge $\mathrm{NH}_{2}{ }^{+}$, which was expected to interact with gram-negative bacterial surface that predominantly consisted of anionic components, such as lipopolysaccharides, phospholipids, and lipoproteins. Indeed, previous studies have indicated that this charge interaction could disrupt the organization of the outer membrane in bacteria and increase its permeability $[11,14]$. Therefore, it could be suggested that the inhibitory activity of CLCG to the Bcc strains is mainly comes from its positive charge.

\subsection{X-ray Diffraction Analysis}

The change of chitosan structure before and after cross-linking with glutaraldehyde was investigated by means of powder XRD, which is a proven tool to study crystal lattice arrangements and yields very useful information on degree of sample crystallinity. The XRD pattern of chitosan in this study displayed two sharp diffraction peaks at $2 \theta=10^{\circ}$ and $20^{\circ}$, revealing the high crystallinity of chitosan. This result is consistent with the result of a number of previous studies [22,32,33], which reported the typical X-ray diffraction pattern of chitosan. The high crystallinity is due to the chitosan structure while plenty of hydroxyl and amino groups could form strong intermolecular and intramolecular hydrogen bonds [33]. In addition, the structure of chitosan molecules has certain regularity. As a result, chitosan molecules could form crystalline regions very easily.

However, as regards CLCG, the characteristic peaks at $2 \theta=10^{\circ}$ and $20^{\circ}$ disappeared, and a very weak and broad peak centered at $2 \theta=15^{\circ}$ appeared (Figure 4). This difference in XRD patterns between chitosan and CLCG should be attributed to the cross-linking reaction between chitosan and glutaraldehyde. In agreement with the result of previous studies $[32,33,39]$, the crystallinity of CLCG decreases after crosslinking with glutaraldehyde. This could be attributed to the deformation of the strong hydrogen bond in original chitosan due to the substitution of hydroxyl and amino groups, which efficiently destroyed the regularity of the packing of the original chitosan chains and resulted in the formation of amorphous CLCG. 
Figure 4. X-ray diffractograms of (a) chitosan and (b) cross-linked chitosan-glutaraldehyde (CLCG).
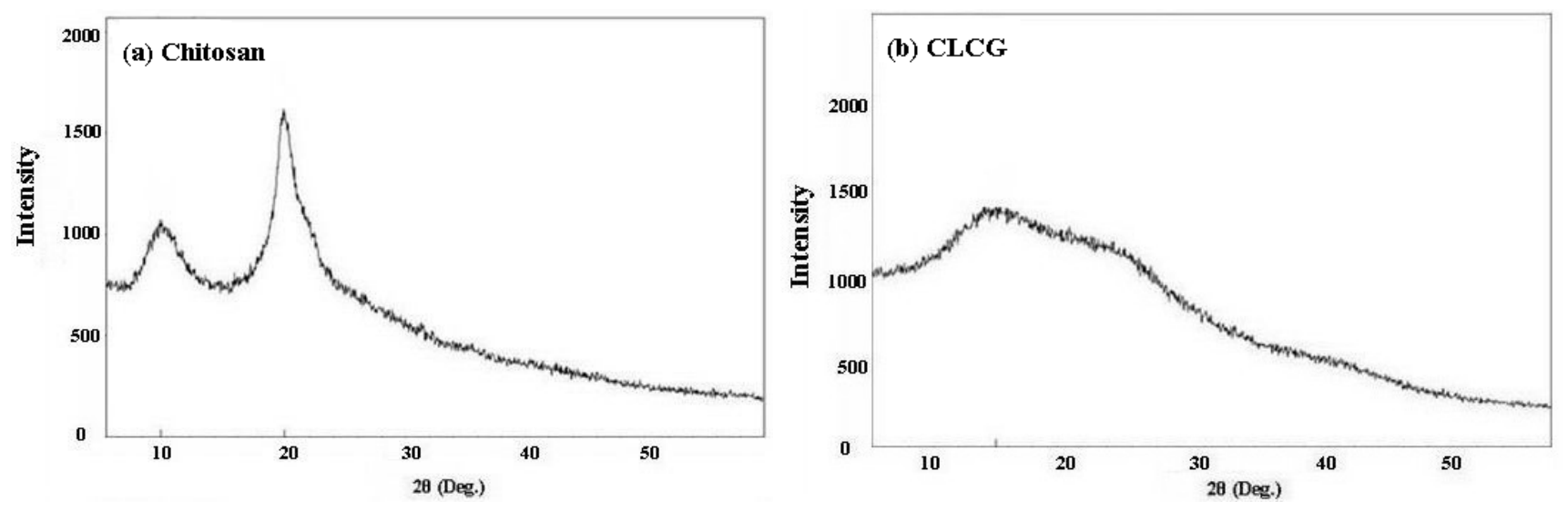

Recently, Mohamed and Fahmy [54] revealed that the incorporation of hydrophilic cross-linker into chitosan allowed the synthesis of hydrogels with higher hydrophilicity, with greater positive charge density and with higher antimicrobial activities, which may explain the result that CLCG had a better antibacterial activity against the Bcc strains compared to the solid chitosan. As mentioned above, the antibacterial mechanism of CLCG may be mainly attributed to the interaction between positive charged CLCG molecules and negatively charged bacterial cell membranes. It suggests that the greater the number of cationized groups, the higher the antibacterial activity. Furthermore, the decrease in intermolecular hydrogen bonds of CLCG could result in the increase of its solubility, which is able to facilitate the penetration of CLCG into the cells of bacteria, thereby preventing the transformation of DNA to RNA to obtain a higher antibacterial activity. In addition, the antibacterial activity of the amorphous CLCG against the Bcc strains may partially due to its ability to chelate metal ions that bind to nutrients essential to bacterial growth.

\subsection{Elemental Analysis of CLCG}

Results from this study indicated that there was a difference in the elements between chitosan and CLCG. Indeed, the element analysis indicated that CLCG had a composition of $33.5 \%(\mathrm{w} / \mathrm{w})$ carbon, $6.5 \%(\mathrm{w} / \mathrm{w})$ hydrogen and $1.6 \%(\mathrm{w} / \mathrm{w})$ nitrogen. In agreement with the result of Gupta et al. [41], this study indicated that the contents of carbon, hydrogen and nitrogen in CLCG were lower than the corresponding contents in chitosan, which reduced by $17.2 \%, 7.9 \%$ and $77.4 \%$, respectively (Table 2 ). In contrast, Oyrton et al. [39] found that the content of carbon and hydrogen percentages is followed by an increase of glutaraldehyde in the chitosan sequence of modified polymers, while the amount of nitrogen decreases with the increasing degree of glutaraldehyde in this series of samples. Therefore, this differential result may be attributed to the difference in the degree of crosslinking.

Table 2. Elementary analysis of chitosan and cross-linked chitosan-glutaraldehyde (CLCG).

\begin{tabular}{cccc}
\hline \multirow{2}{*}{ Substance } & \multicolumn{3}{c}{ Element $(\%, w / w)$} \\
\cline { 2 - 4 } & Carbon & Hydrogen & Nitrogen \\
\hline Chitosan & 40.47 & 7.08 & 7.21 \\
CLCG & 33.50 & 6.52 & 1.63 \\
\hline
\end{tabular}


From this result, it could be supposed that the cross-linking reaction happened and new functional groups were produced, while the antibacterial activity of CLCG against the Bcc strains is associated with the decrease in the contents of carbon, hydrogen and nitrogen. Indeed, the reduction in the elements could be mainly attributed to the cross-linking reaction between the aldehyde groups of glutaraldehyde and amino groups of chitosan, which resulted in the formation of amorphous CLCG with higher hydrophilicity. Furthermore, as a matter of fact, the presence of $\mathrm{H}$ and $\mathrm{O}$ from moisture is also associated to the decrease in contents of the elements, in particular, carbon, and nitrogen. In addition, the loosely packed structure has obviously played an important role in the antibacterial activity of CLCG against the Bcc strains, which has been described as above.

\subsection{Thermo Gravimetric Analysis of CLCG}

Thermo gravimetric analysis for the experimental samples of chitosan and CLCG were illustrated in Figure 5 and the corresponding thermal degradation values were displayed in Table 3. This result revealed that the thermal degradation curves of chitosan and CLCG were dependent on the temperature, which is consistent with the above result that the antibacterial activity of CLCG was slightly changed when it was treated under different temperature. In general, this result revealed that the thermal stability of CLCG is lower than that of chitosan regardless of the temperature. The weight loss of CLCG is $368.1 \%-384.7 \%$ higher than that of chitosan in the first stage $\left(100{ }^{\circ} \mathrm{C}-200{ }^{\circ} \mathrm{C}\right), 96.8 \%-384.7 \%$ higher than that of chitosan in the second stage $\left(200{ }^{\circ} \mathrm{C}-300{ }^{\circ} \mathrm{C}\right)$ and $25.7 \%-96.8 \%$ higher than that of chitosan in the third stage $\left(300^{\circ} \mathrm{C}-800^{\circ} \mathrm{C}\right)$ of thermal degradation.

Table 3. Thermal analysis of chitosan and cross-linked chitosan-glutaraldehyde (CLCG) under $\mathrm{N}_{2}$ air atmosphere.

\begin{tabular}{ccc}
\hline \multirow{2}{*}{ Temperature $\left({ }^{\circ} \mathbf{C}\right)$} & \% of weight loss (heating rate $\left.20{ }^{\circ} \mathbf{C} / \mathbf{m i n}\right)$ \\
\cline { 2 - 3 } & Chitosan & CLCG \\
\hline 100 & 10.13 & 47.42 \\
300 & 11.25 & 54.53 \\
400 & 35.06 & 68.99 \\
500 & 59.27 & 76.59 \\
600 & 60.08 & 87.80 \\
700 & 68.39 & 89.07 \\
800 & 70.21 & 89.50 \\
\hline
\end{tabular}

Previous studies have indicated that the weight loss in the first stage may be mainly attributed to water loss, while in the second and third stage may be mainly due to the breakage of main chain and decomposition of glucose units, respectively $[32,55]$. Interestingly, results from this study indicated that the weight loss of CLCG at $800{ }^{\circ} \mathrm{C}$ is $30.2 \%$ higher than that of CLCG at $300{ }^{\circ} \mathrm{C}$, while the weight loss of chitosan at $800{ }^{\circ} \mathrm{C}$ is $103.7 \%$ higher than that of chitosan at $300{ }^{\circ} \mathrm{C}$, revealing that CLCG is more stable in the glucose units under high temperature compared to chitosan. Therefore, it could be suggested that the weight loss of CLCG may be mainly due to the loss of water. 
Figure 5. Thermo gravimetric analysis of (a) chitosan and (b) cross-linked chitosan-glutaraldehyde (CLCG).
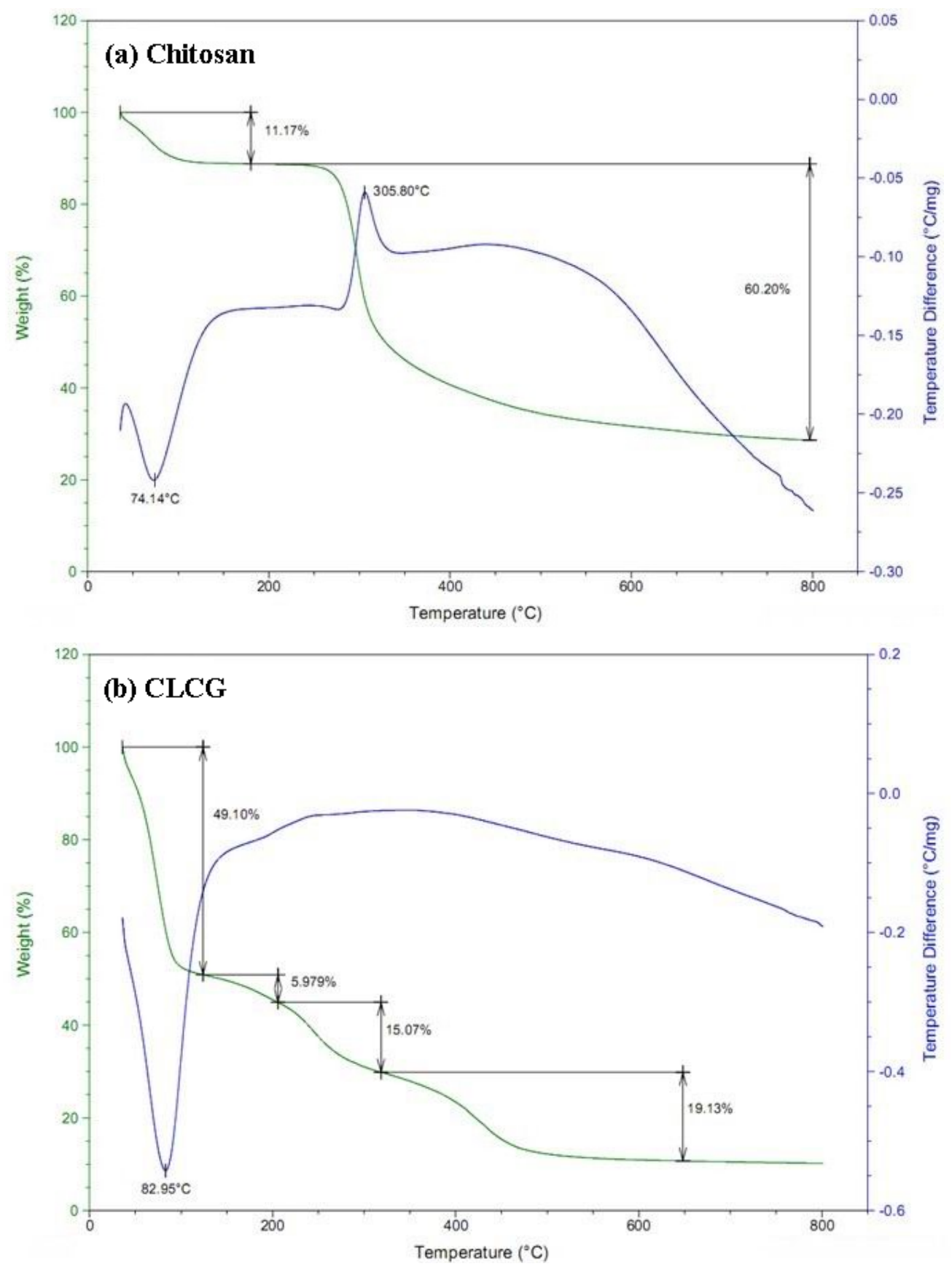

The result from this study indicated that CLCG has a high water content, while solid chitosan used in this study was unable to be dissolved in water. This revealed that the antibacterial activity of CLCG may be due to its high hydrophilicity. Furthermore, Zhao et al. [55] found that the drop in the temperature of water loss and the increase in the moisture content were related to the increase in pore spaces resulting from crosslinking. Therefore, the increased antibacterial activity of CLCG was able to be attributed to the difference in the thermal degradation curve between chitosan and CLCG. In addition, this result of the thermal degradation curve also indicated that new functional groups maybe have been produced due to the cross-linking reaction, which is consistent with the results of SEM, FTIR spectra, XRD pattern and elemental analysis. 


\section{Experimental Section}

\subsection{Chitosan, Glutaraldehyde and Bacteria}

Chitosan (molecular weight of $1129 \mathrm{KDa}$, degree of $N$-deacetylation no less than $85 \%$, practical grade, from crab shells) was obtained from Sigma-Aldrich (St. Louis, MO, USA), while glutaraldehyde was purchased as a $25 \%$ stock from Sigma Chemical Co. (St. Louis, MO, USA). In addition, the tested Bcc strains were shown in Table 1, which have been isolated in our previous studies and deposited in the culture collection of the Institute of Biotechnology, Zhejiang University, China.

\subsection{Cross-Linking of Chitosan and Glutaraldehyde}

CLCG was synthesized by dissolving $2.0 \mathrm{~g}$ of chitosan into $50.0 \mathrm{~mL}$ of $5.0 \%$ acetic acid and then adding $15.0 \mathrm{~mL} 25 \%$ glutaraldehyde into this chitosan solution to form a water gel after $24 \mathrm{~h}$ of stirring at room temperature by using a magnetic stirrer, while the non cross-linked glutaraldehyde was removed by washing the cross-linked complex more than eight times with double distilled water. The obtained CLCG was then lyophilised and stored at $-70^{\circ} \mathrm{C}$.

\subsection{Antibacterial Activity}

The antibacterial activity of CLCG at room temperature $\left(20^{\circ} \mathrm{C}\right)$ against nine different Bcc strains was performed in LB medium according to the method of stainless steel cylinders as described by Li et al. [56]. In brief, the final concentration of the Bcc strains in LB medium was $10^{8} \mathrm{CFU} / \mathrm{mL}$, while each stainless steel cylinder was covered with a thin layer of CLCG (about $0.05 \mathrm{~g}$ ). Each treatment was replicated using five stainless steel cylinders, and the experiment was performed three times.

The effect of temperature in the antibacterial activity of CLCG was carried out by incubating CLCG in sterile water of $40{ }^{\circ} \mathrm{C}, 70{ }^{\circ} \mathrm{C}$ and $100{ }^{\circ} \mathrm{C}$ for $2 \mathrm{~h}$, respectively, and then washing CLCG twice with distilled water. In addition, the effect of time in the antibacterial activity of CLCG was performed by incubating CLCG and bacteria for 12 h, 24 h, and 48 h, respectively. The antibacterial activity of CLCG under different environments was determined as described above.

\subsection{Determination of Cross-Linking Degree}

The cross-linking degree of CLCG is representative of a ratio of mass of cross-linked state of CLCG to the whole mass of CLCG. The cross-linking degree of the obtained CLCG was determined by dissolving CLCG into $2.0 \%$ acetic acid for $24 \mathrm{~h}$ and then measuring the dry weight of pre and post-dissolved CLCG as described by Mitra et al. [26].

\subsection{Scan Electron Microscope}

The CLCG sample was washed twice with $0.1 \mathrm{M}$ phosphate buffer solution ( $\mathrm{pH} 7.0, \mathrm{PBS})$ and fixed with $2.5 \%(\mathrm{v} / \mathrm{v})$ glutaraldehyde in $0.1 \mathrm{M}$ PBS. Then the CLCG sample was postfixed with $1 \%(\mathrm{w} / \mathrm{v})$ OsO4 in $0.1 \mathrm{M}$ PBS for $1 \mathrm{~h}$ at room temperature and washed three times with the same buffer, dehydrated separately at $4{ }^{\circ} \mathrm{C}$ for $15 \mathrm{~min}$ in a graded series of ethanol solutions $(70,80,90,95$ and 100\%, v/v), then embedded in Epon 812 a low-viscosity embedding medium. The thin section 
specimen was cut with a diamond knife on an Ultracut Ultramicrotome (Super Nova; Reichert-Jung Optische Werke, Wien, Austria) and double-stained with saturated uranyl acetate and lead citrate. As described by Wang et al. [57], the grids were examined by using a JEM-1230 transmission electron microscope (Hitachi, Tokyo, Japan) at an operating voltage of $75 \mathrm{kV}$.

\subsection{Fourier Transform Infrared Spectra}

The samples of chitosan and CLCG were first grounded into fine particles using mortar and pestle. The 1.0-2.0 mg of each sample was then mixed with $200 \mathrm{mg}$ potassium bromide (KBr) which extensively dried in microfuge tubes using a lyophiliser. The mixtures have been dried for an additional $2 \mathrm{~h}$ in the same microfuge tubes. The $\mathrm{KBr}$ based pellets were then compressed into a thin disk by establishing pressure of $(5-10) \times 10^{7} \mathrm{~Pa}$. The ATR measurements were performed using an FTIR spectrometer (VERTEX 70).

Pellets were scanned at $4 \mathrm{~cm}^{-1}$ resolution with 100 scans in the spectral range of $4000-500 \mathrm{~cm}^{-1}$ at room temperature. The sample compartment in the FTIR spectrometer was continuously purged with dry air to prevent water vapor. Analysis of the spectral data was performed as described by Garip et al. [58] by using Grams 32 (Galactic Industries, Salem, NH, USA) software. The spectral range of 4000-500 $\mathrm{cm}^{-1}$ was analyzed. The band positions were measured according to the center of weight and the spectra obtained from the same experimental groups, baseline correction, normalization and the band areas were averaged. The average spectra and normalization process were applied only for visual representation of the differences between chitosan and CLCG, while each original baseline corrected spectrum was taken into consideration for the determination of the spectral parameters and calculation of mean values.

\subsection{X-ray Diffraction Analysis}

The XRD measurements of chitosan and CLCG were recorded as described by Ge et al. [22] and Zhang et al. [59] by using an XPert PRO diffractometer (Holland) with a detector operating under a voltage of $40.0 \mathrm{kV}$ and a current of $30.0 \mathrm{~mA}$ using CuKo radiation. The recording range of $2 \theta$ was $5^{\circ}$ to $60^{\circ}$, and the scanning speed was $6^{\circ} / \mathrm{min}$.

\subsection{Elemental Analysis}

The contents of carbon, hydrogen and nitrogen elements in chitosan and CLCG were determined according to the method of Yao et al. [52], which were performed on a vario microorganic elemental analyzer (CE Intrusment EA1112, Italy).

\subsection{Thermo Gravimetric Analysis}

Thermal decomposition analysis of chitosan and CLCG was carried out as described by Mitra et al. [26] under nitrogen flow $\left(40 \& 60 \mathrm{~mL} / \mathrm{min}\right.$ ) with $\mathrm{ramp} 10^{\circ} \mathrm{C} / \mathrm{min}$ using Universal V4.3A TA instruments. The temperature range is between $0{ }^{\circ} \mathrm{C}$ and $800{ }^{\circ} \mathrm{C}$. 


\subsection{Statics Analysis}

The software STATGRAPHICS Plus, version 4.0 (Copyright Manugistics Inc., Rockville, MD, USA) was used to perform the statistical analysis. Levels of significance $(p<0.05)$ of main treatments and their interactions were calculated by analysis of variance after testing for normality and variance homogeneity.

\section{Conclusions}

Results from this study indicated that the growth of Bcc strains was unaffected by solid chitosan, but was strongly inhibited by the synthesized glutaraldehyde cross-linked chitosan with cross-linking degree of $80.8 \%$ regardless of bacterial species and incubation time. Furthermore, CLCG treated with high temperature showed antibacterial activity against the selected strain 0901 of the Bcc although the inhibitory effects varied with different temperatures. In addition, the differential anti-Bcc activity between chitosan and glutaraldehyde cross-linked chitosan may be mainly due to the difference in their physical-chemical properties, which has been determined based on SEM observation, FTIR spectra, $\mathrm{X}$-ray diffraction pattern, as well as elemental and thermo gravimetric analysis. Overall, this study revealed that the use of glutaraldehyde cross-linked chitosan as a potential antibacterial agent seems to be promising in reducing the risk of $\mathrm{CF}$ patients.

\section{Acknowledgments}

This project was supported by Zhejiang Provincial Natural Science Foundation of China (R13C140001; Y3090150; LQ12C14003), National Natural Science Foundation of China (31271700), Zhejiang Provincial Project (2010R10091), the Fundamental Research Funds for the Central Universities, the Agricultural Ministry of China (nyhyzx 201003029; 201003066; 201303015), State Education Ministry and Key Subject Construction Program of Zhejiang for Modern Agricultural Biotechnology and Crop Disease Control (2010DS700124-KF1101; 2010DS700124-KF1203).

\section{Conflict of Interest}

The authors declare no conflict of interest.

\section{References}

1. Mahenthiralingam, E.; Baldwin, A.; Dowson, C.G. Burkholderia cepacia complex bacteria: Opportunistic pathogens with important natural biology. J. Appl. Microbiol. 2008, 104, 1539-1551.

2. Vanlaere, E.; Lipuma, J.J.; Baldwin, A.; Henry, D.; De Brandt, E.; Mahenthiralingam, E.; Speert, D.; Dowson, C.; Vandamme, P. Burkholderia latens sp. nov., Burkholderia diffusa sp. nov., Burkholderia arboris sp. nov., Burkholderia seminalis sp. nov. and Burkholderia metallica sp. nov., novel species within the Burkholderia cepacia complex. Int. J. Syst. Evol. Microbiol. 2008, 58, 1580-1590. 
3. Vanlaere, E.; Baldwin, A.; Gevers, D.; Henry, D.; Brandt, E.D.; LiPuma, J.J. Taxon K, a complex within the Burkholderia cepacia complex, comprises at least two novel species: Burkholderia contaminans sp. nov. and Burkholderia lata sp. nov. Int. J. Syst. Evol. Microbiol. 2009, 59, 102-111.

4. Fang, Y.; Lou, M.M.; Li, B.; Xie, G.L.; Wang, F.; Zhang, L.X.; Luo, Y.C. Characterization of Burkholderia cepacia complex from cystic fibrosis patients in China and their chitosan susceptibility. World J. Microbiol. Biotechnol. 2010, 26, 443-450.

5. Ibrahim, M.; Fang, Y.; Lou, M.M.; Xie, G.L.; Li, B.; Zhu, B.; Zhang, G.Q.; Liu, H.; Wareth, A. Copper as an antibacterial agent for human pathogenic multidrug resistant Burkholderia cepacia complex bacteria. J. Biosci. Bioeng. 2011, 112, 570-576.

6. Ibrahim, M.; Tang, Q.M.; Shi, Y.; Almoneafy, A.; Fang, Y.; Xu, L.; Li, W.; Li, B.; Xie, G.L. Diversity of potential pathogenicity and biofilm formation among Burkholderia cepacia complex water, clinical, and agricultural isolates in China. World J. Microbiol. Biotechnol. 2012, 28, 2113-2123.

7. Fang, Y.; Xie, G.L.; Lou, M.M.; Li, B.; Ibrahim, M. Diversity analysis of Burkholderia cepacia complex in the water bodies of west lake, Hangzhou, China. J. Microbiol. 2011, 49, 309-314.

8. Li, B.; Fang, Y.; Zhang, G.Q.; Yu, R.R.; Lou, M.M.; Xie, G.L.; Wang, Y.L.; Sun, G.C. Molecular characterization of Burkholderia cepacia complex isolates causing bacterial fruit rot of apricot. Plant Pathol. J. 2010, 26, 223-230.

9. Lou, M.M.; Fang, Y.; Zhang, G.Q.; Xie, G.L.; Zhu, B.; Muhammad, I. Diversity of Burkholderia cepacia complex from the moso bamboo (Phyllostachys edulis) rhizhosphere soil. Curr. Microbiol. 2011, 62, 650-658.

10. Zhang, L.X.; Xie, G.L. Diversity and distribution of Burkholderia cepacia complex in the rhizosphere of rice and maize. FEMS Microbiol. Lett. 2007, 266, 231-235.

11. Lou, M.M.; Zhu, B.; Ibrahim, M.; Li, B.; Xie, G.L.; Li, H.Y. Antibacterial activity and mechanism of action of chitosan solution against apricot fruit rot pathogen Burkholderia seminalis. Carbohydr. Res. 2011, 346, 1294-1301.

12. Hadrami, A.E.; Adam, L.R.; Hadrami, I.E.; Daayf, F. Chitosan in plant protection. Mar. Drugs 2010, 8, 968-987.

13. Kumirska, J.; Czerwicka, M.; Kaczyński, Z.; Bychowska, A.; Brzozowski, K.; Thöming, J.; Stepnowski, P. Application of spectroscopic methods for structural analysis of chitin and chitosan. Mar. Drugs 2010, 8, 1567-1636.

14. Li, B.; Wang, X.; Chen, R.X.; Huangfu, W.G.; Xie, G.L. Antibacterial activity of chitosan solution against Xanthomonas pathogenic bacteria isolated from Euphorbia pulcherrima. Carbohydr. Polym. 2008, 72, 287-292.

15. Badawy, M.E.I. Effect of depolymerization degree of the natural biopolymer chitosan on some plant pathogenic bacteria and fungi. J. Pest Control Environ. Sci. 2007, 15, 69-85.

16. Badawy, M.E.I. Structure and antimicrobial activity relationship of quaternary $N$-alkyl chitosan derivatives against some plant pathogens. J. Appl. Polym. Sci. 2010, 117, 960-969.

17. Li, B.; Liu, B.P.; Su, T.; Wang, F.; Tang, Q.M.; Fang, Y.; Xie, G.L.; Sun, G.C. Effect of chitosan solution on the inhibition of Pseudomonas fluorescens causing bacterial head rot of broccoli. Plant Pathol. J. 2010, 26, 189-193. 
18. Li, B.; Su, T.; Chen, X.L.; Liu, B.P.; Zhu, B.; Fang, Y.; Xie, G.L.; Wang, G.F.; Wang, Y.L.; Sun, G.C. Effect of chitosan solution on the bacterial septicemia disease of Bombyx mori (Lepidoptera: Bombycidae) caused by Serratia marcescens. Appl. Entomol. Zool. 2010, 45, 145-152.

19. Li, B.; Yu R.R.; Liu, B.P.; Tang, Q.M.; Zhang, G.Q.; Wang, Y.L.; Xie, G.L.; Sun, G.C. Characterization and comparison of Serratia marcescens isolated from edible cactus and from silkworm for virulence potential and chitosan susceptibility. Braz. J. Microbiol. 2011, 42, 96-104.

20. Khoushab, F.; Yamabhai, M. Chitin research revisited. Mar. Drugs 2010, 8, 1988-2012.

21. Trimukhe, K.D.; Varma, A.J. Metal complexes of crosslinked chitosans: Correlations between metal ion complexation values and thermal properties. Carbohydr. Polym. 2009, 75, 63-70.

22. Ge, H.C.; Chen, H.; Huang, S.Y. Microwave preparation and properties of $O$-crosslinked maleic acyl chitosan adsorbent for $\mathrm{Pb}^{2+}$ and $\mathrm{Cu}^{2+}$. J. Appl. Polym. Sci. 2012, 125, 2716-2723.

23. Liu, J.H.; Wang, Q.; Wang, A.Q. Synthesis and characterization of chitosan-g-poly(acrylic acid)/sodium humate superabsorbent. Carbohydr. Polym. 2007, 70, 166-173.

24. Chen, J.K.; Yeh, C.H.; Wang, L.C.; Liou, T.H.; Shen, C.R.; Liu, C.L. Chitosan, the marine functional food, is a potent adsorbent of humic acid. Mar. Drugs 2011, 9, 2488-2498.

25. Guo, L.; Liu, G.; Hong, R.Y.; Li, H.Z. Preparation and characterization of chitosan poly(acrylic acid) magnetic microspheres. Mar. Drugs 2010, 8, 2212-2222.

26. Mitra, T.; Sailakshmi, G.; Gnanamani, A.; Manda, A.B. Preparation and characterization of malonic acid cross-linked chitosan and collagen 3D scaffolds: An approach on non-covalent interactions. J. Mater. Sci. 2012, 23, 1309-1321.

27. Yu, Q.; Song, Y.N.; Shi, X.M.; Xu, C.Y.; Bin, Y.Z. Preparation and properties of chitosan derivative/poly(vinyl alcohol) blend film crosslinked with glutaraldehyde. Carbohydr. Polym. 2011, 84, 465-470.

28. Zhao, J.H.; Han, W.Q.; Chen, H.D.; Tu, M.; Zeng, R.; Shi, Y.F.; Cha, Z.G.; Zhou, C.R. Preparation, structure and crystallinity of chitosan nano-fibers by a solid-liquid phase separation technique. Carbohydr. Polym. 2011, 83, 1541-1546.

29. Jabli, M.; Baouab, M.H.V.; Sintes-Zydowicz, N.; Hassine, B.B. [Dye Molecules/Copper(II)/ Macroporous Glutaraldehyde-Chitosan] Microspheres complex: Surface characterization, kinetic, and thermodynamic investigations. J. Appl. Polym. Sci. 2012, 123, 3412-3424.

30. Suguna, M.; Kumar, N.S.; Reddy, A.S.; Boddu, V.M.; Krishnaiah, A. Biosorption of lead (II) from aqueous solution on glutaraldehyde cross-linked chitosan beads. Can. J. Chem. Eng. 2011, 89, 833-843.

31. Varmaa, A.J.; Deshpande, S.V.; Kennedy, J.F. Metal complexation by chitosan and its derivatives: A review. Carbohydr. Polym. 2004, 55, 77-93.

32. Tripathi, S.; Mehrotra, G.K.; Dutta, P.K. Physicochemical and bioactivity of cross-linked chitosan-PVA film for food packaging applications. Int. J. Biol. Macromol. 2009, 45, 372-376.

33. Saita, K.; Nagaoka, S.; Shirosaki, T.; Horikawa, M.; Matsuda, S.; Ihara, H. Preparation and characterization of dispersible chitosan particles with borate crosslinking and their antimicrobial and antifungal activity. Carbohydr. Res. 2012, 349, 52-58. 
34. Wiarachai, O.; Thongchul, N.; Kiatkamjornwong, S.; Hoven, V.P. Surface-quaternized chitosan particles as an alternative and effective organic antibacterial material. Colloid Surface B 2012, 92, $121-129$.

35. Zhang, Z.T.; Chen, L.; Ji, J.M.; Huang, Y.L.; Chen, D.H. Antibacterial properties of cotton fabrics treated with chitosan. Text. Res. J. 2003, 73, 1103-1106.

36. Wang, C.C.; Yang, F.L.; Zhang, H.M. Fabrication of non-woven composite membrane by chitosan coating for resisting the adsorption of proteins and the adhesion of bacteria. Sep. Purif. Technol. 2010, 75, 358-365.

37. Mcconnell, E.L.; Murdan, S.; Basit, A.W. An investigation into the digestion of CHITOSAN (noncrosslinked and crosslinked) by human colonic bacteria. J. Pharm. Sci. 2008, 97, 3820-3829.

38. Argüelles-Monal, W.; Goycoolea, F.M.; Peniche, C.; Higuera-Ciapara, I. Rheological study of the chitosan/glutaraldehyde chemical gel system. Polym. Gels Networks 1998, 6, 429-440.

39. Oyrton, A.C.; Monteiro, J.; Claudio, A. Some studies of crosslinking chitosan-glutaraldehyde interaction in a homogeneous system. Int. J. Biol. Macromol. 1999, 26, 119-128.

40. Ignatova, M.; Manolova, N.; Markova, N.; Rashkov, I. Electrospun non-woven nanofibrous hybrid mats based on chitosan and PLA for wound-dressing applications. Macromol. Biosci. 2009, 9, $102-111$.

41. Gupta, K.C.; Jabrail, F.H. Glutaraldehyde and glyoxal cross-linked chitosan microspheres for controlled delivery of centchroman. Carbohydr. Res. 2006, 341, 744-756.

42. Gonçalves, V.L.; Laranjeira, M.C.M.; Fávere, V.T. Effect of crosslinking agents on chitosan microspheres in controlled release of diclofenac sodium. Polímeros 2005, 15, 6-12.

43. Dini, E.; Alexandridou, S.; Kiparissides, C. Synthesis and characterization of cross-linked chitosan microspheres for drug delivery applications. J. Microencapsul. 2003, 20, 375-385.

44. Rodrigues, D.S.; Mendes, A.A.; Adriano, W.S.; Gonçalves, L.R.B.; Giordano, R.L.C. Multipoint covalent immobilization of microbial lipase on chitosan and agarose activated by different methods. J. Mol. Catal. B 2008, 51, 100-109.

45. Knaul, J.Z.; Hudson, S.M.; Creber, K.A.M. Crosslinking of chitosan fibers with dialdehydes: Proposal of a new reaction mechanism. J. Polym. Sci. Pol. Phys. 1999, 37, 1079-1094.

46. Trimukhe, K.D.; Varma, A.J. A morphological study of heavy metal complexes of chitosan and crosslinked chitosans by SEM and WAXRD. Carbohydr. Polym. 2008, 71, 698-702.

47. Mirzaei, B.E.; Ramazani, S.A.A.; Shafiee, M.; Danaei, M. Studies on glutaraldehyde crosslinked chitosan hydrogel properties for drug delivery systems. Int. J. Polym. Mater. 2013, 62, 605-611.

48. Wang, L.Y.; Gu, Y.H.; Zhou, Q.Z.; Ma, G.H.; Wan, Y.H.; Su, Z.G. Preparation and characterization of uniform-sized chitosan microspheres containing insulin by membrane emulsification and a two-step solidification process. Colloids Surfaces B 2006, 50, 126-135.

49. Kulkarni, V.H.; Kulkarni, P.V.; Keshavayya, J. Glutaraldehyde-crosslinked chitosan beads for controlled release of diclofenac sodium. J. Appl. Polym. Sci. 2007, 103, 211-217.

50. Fang, Y.; Hu, D.D. Cross-linking of chitosan with glutaraldehyde in the presence of citric acid-A new gelling system. Chin. J. Polym. Sci. 1999, 17, 551-556.

51. Aranaz, I.; Mengíbar, M.; Harris, R.; Paños, I.; Miralles, B.; Acosta, N.; Galed, G.; Heras, Á. Functional characterization of chitin and chitosan. Curr. Chem. Biol. 2009, 3, 203-230. 
52. Yao, W.J.; Jiao, Y.; Luo, J.; Du, M.Z.; Li, Z. Practical synthesis and characterization of mannose-modified chitosan. Int. J. Biol. Macromol. 2012, 50, 821-825.

53. Ramachandran, S.; Nandhakumar, S.; Dhanaraju, M.D. Formulation and characterization of glutaraldehyde cross-linked chitosan biodegradable microspheres loaded with famotidine. Trop. J. Pharm. Res. 2011, 10, 309-316.

54. Mohamed, N.A.; Fahmy, M.M. Synthesis and antimicrobial activity of some novel cross-linked chitosan hydrogels. Int. J. Mol. Sci. 2012, 13, 11194-11209.

55. Zhao, X.F.; Li, Z.J.; Wang, L.; Lai, X.J. Synthesis, characterization, and adsorption capacity of crosslinked starch microspheres with $N, N^{\prime}$-methylene Bisacrylamide. J. Appl. Polym. Sci. 2008, 109, 2571-2575.

56. Li, B.; Xu, L.H.; Lou, M.M.; Li, F.; Zhang, Y.D.; Xie, G.L. Isolation and characterization of antagonistic bacteria against bacterial leaf spot of Euphorbia pulcherrima. Lett. Appl. Microbiol. 2008, 46, 450-455.

57. Wang, Y.L.; Li, L.P.; Li, B.; Wu, G.X.; Tang, Q.M.; Muhammad, I.; Liu, H.; Xie, G.L.; Sun, G.C. Action of chitosan against Xanthomonas pathogenic bacteria isolated from Euphorbia pulcherrima. Molecules 2012, 17, 7028-7041.

58. Garip, S.; Cetin, G.A.; Severcan, F. Use of fourier transforms infrared spectroscopy for rapid comparative analysis of Bacillus and Micrococcus isolates. Food Chem. 2009, 113, 1301-1307.

59. Zhang, N.Y.; Shen, Y.G.; Li, X.Q.; Cai, S.J.; Liu, M.Z. Synthesis and characterization of thermo and $\mathrm{pH}$-sensitive poly(vinyl alcohol)/poly ( $N, N$-diethylacrylamide-co-itaconic acid) semi-IPN hydrogels. Biomed. Mater. 2012, 7, 1748-6041.

(C) 2013 by the authors; licensee MDPI, Basel, Switzerland. This article is an open access article distributed under the terms and conditions of the Creative Commons Attribution license (http://creativecommons.org/licenses/by/3.0/). 